\title{
Richard Poulin
}

Sociologue, professeur titulaire, département de sociologie et d'anthropologie, Université d'Ottawa.

(2009)

\section{"Apparence, hypersexualisation et pornographie."}

Un document produit en version numérique par Jean-Marie Tremblay, bénévole, professeur de sociologie retraité du Cégep de Chicoutimi

Courriel: jean-marie_tremblay@uqac.ca

Site web pédagogique : $h+t p: / / w w w . u q a c . c a / j m t-s o c i o l o g u e /$

Dans le cadre de: "Les classiques des sciences sociales"

Une bibliothèque numérique fondée et dirigée par Jean-Marie Tremblay, professeur de sociologie au Cégep de Chicoutimi

Site web: http://classiques.uqac.ca/

Une collection développée en collaboration avec la Bibliothèque

Paul-Émile-Boulet de l'Université du Québec à Chicoutimi

Site web: http://bibliotheque.uqac.cal 


\section{Politique d'utilisation de la bibliothèque des Classiques}

Toute reproduction et rediffusion de nos fichiers est interdite, même avec la mention de leur provenance, sans l'autorisation formelle, écrite, du fondateur des Classiques des sciences sociales, Jean-Marie Tremblay, sociologue.

Les fichiers des Classiques des sciences sociales ne peuvent sans autorisation formelle:

- être hébergés (en fichier ou page web, en totalité ou en partie) sur un serveur autre que celui des Classiques.

- servir de base de travail à un autre fichier modifié ensuite par tout autre moyen (couleur, police, mise en page, extraits, support, etc...),

Les fichiers (.html, .doc, .pdf, .rtf, .jpg, .gif) disponibles sur le site Les Classiques des sciences sociales sont la propriété des Classiques des sciences sociales, un organisme à but non lucratif composé exclusivement de bénévoles.

Ils sont disponibles pour une utilisation intellectuelle et personnelle et, en aucun cas, commerciale. Toute utilisation à des fins commerciales des fichiers sur ce site est strictement interdite et toute rediffusion est également strictement interdite.

L'accès à notre travail est libre et gratuit à tous les utilisateurs. C'est notre mission.

Jean-Marie Tremblay, sociologue

Fondateur et Président-directeur général, LES CLASSIQUES DES SCIENCES SOCIALES. 
Cette édition électronique a été réalisée par Jean-Marie Tremblay, bénévole, professeur de sociologie au Cégep de Chicoutimi à partir de:

\section{Richard POULIN}

"Apparence, hypersexualisation et pornographie".

Un article publié dans la revue Nouveaux cahiers du socialisme, n 1, hiver 2009, pp. 227-245. Numéro intitulé : "Les classes sociales aujourd'hui."

[Autorisation formelle accordée par l'auteur le 13 septembre 2011 de diffuser ce texte dans Les Classiques des sciences sociales.]

Courriel : Richard.Poulin@uottawa.ca

Polices de caractères utilisée: Comic Sans, 12 points.

Édition électronique réalisée avec le traitement de textes Microsoft Word 2008 pour Macintosh.

Mise en page sur papier format : LETTRE US, $8.5^{\prime \prime} \times 11^{\prime \prime}$.

Édition numérique réalisée le 4 septembre 2011 à Chicoutimi, Ville de Saguenay, Royaume du Saguenay, Québec. 


\section{Richard Poulin}

Sociologue, professeur titulaire, département de sociologie et d'anthropologie, Université d'Ottawa.

\section{"Apparence, hypersexualisation et pornographie".}

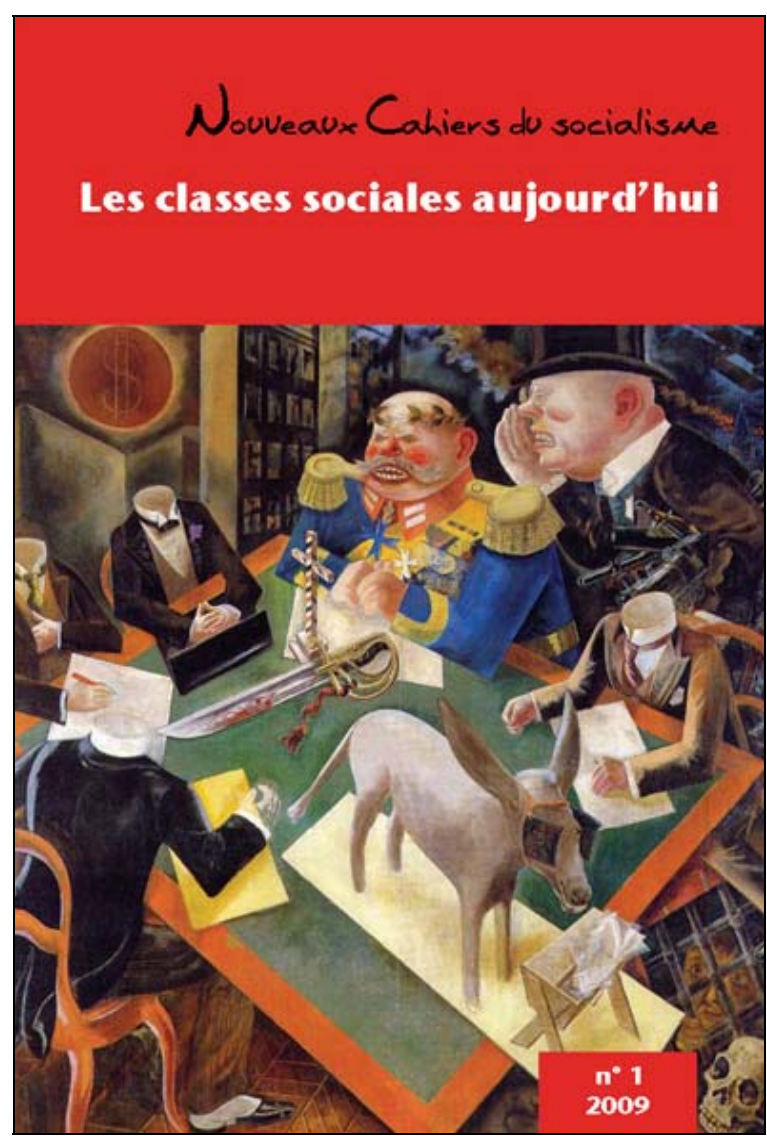

Un article publié dans la revue Nouveaux cahiers du socialisme, n 1, hiver 2009, pp. 227-245. Numéro intitulé: "Les classes sociales aujourd'hui." 


\section{Table des matières}

Introduction

La presse féminine

La publicité « porno chic »

La mode hypersexualisée

La sexualisation publique et la pornographisation

La tyrannie du nouvel ordre sexuel

La saturation sexuelle et sexiste de l'espace public

De l'intimité surexposée

L'influence sociale pornographique

Pouvoirs 
Richard Poulin

Sociologue, professeur titulaire, département de sociologie et d'anthropologie, Université d'Ottawa.

"Apparence, hypersexualisation et pornographie".

Un article publié dans la revue Nouveaux cahiers du socialisme, n 1, hiver 2009, pp. 227-245. Numéro intitulé : "Les classes sociales aujourd'hui."

\section{Introduction}

Séduire, c'est mourir comme réalité et se produire comme leurre

Jean Baudrillard 1

\section{Retour à la table des matières}

Faites votre cinéma $X X X$ ! Silence, on jouit! Guide du top dix des films érotiques. Les films pornographiques peuvent alimenter votre vie sexuelle! Préparons le pop-corn et le lubrifiant! Cours de danse sexy pour booster la séductrice en nous! Fais-moi mal chéri! Festival de la branlette!

Ce ne sont pas des titres coiffant des DVD pornographiques ou des articles de magazines pour hommes! Ces titres sont tirés du magazine FA (Femmes d'aujourd'hui, mai 2008), publié par TVA Publications, une compagnie de la multinationale Quebecor qui possède également la principale chaîne privée de la télévision québécoise. FA racole pour la pornographie en tentant de convaincre les femmes que celle-ci est glamour, sexy et enrichissante. Ce magazine a le soutien financier du

1 Jean Baudrillard, De la séduction, Paris, Galilée, 1979, p. 98. 
gouvernement canadien, par l'entremise du Programme d'aide aux publications et du Fonds du Canada pour les magazines, pour ses envois postaux et ses coûts rédactionnels.

Le groupe Quebecor est propriétaire de Vidéotron, l'une des principales compagnies de câblodistribution et de connexion à Internet. Par conséquent, Quebecor s'avère l'un des principaux diffuseurs de la pornographie à la télévision, par Internet et par la [228] téléphonie sans fil. Le groupe encourage ouvertement sa diffusion dans ses nombreux magazines et ses hebdomadaires gratuits. La multinationale contrôle également les principaux portails commerciaux québécois de I'Internet, dont un moteur de recherche, des agences de rencontre ainsi que des réseaux de communautés en ligne.

Les magazines comme FA sont l'aboutissement d'une tendance à l'oeuvre depuis les années 1990, soit depuis que la pornographie s'ébat avec succès hors de son ghetto, en imposant ses normes.

\section{La presse féminine}

\section{Retour à la table des matières}

La presse féminine, qui était traditionnellement sentimentale, est devenue «sexuelle»; le «si on se fiançait» a évolué pour devenir « comment mettre un homme dans son lit» (Isa, mars 2001). Elle fait nettement écho à l'imagerie pornographique. L'acte sexuel est dissocié des sentiments, ce qui, on le sait, est la trame même du discours pornographique: "Instant sexe, le guide de l'amour rapido-incognito", titre Vingt ans (mai 2000), un magazine consommé par les adolescentes et même les préadolescentes.

Il s'agit non seulement de «booster son plaisir 》 (Bien dans ma vie!, été 2005) et de croire que tout ce qui est pornographique est hot, mais surtout de réguler la sexualité féminine autour de l'idée de la performance sexuelle. Les femmes et les adolescentes doivent absolument vivre une sexualité épanouie ${ }^{2}$. Cette dernière exige à la fois

2 Caroline Moulin, Féminités adolescentes, Rennes, Presses universitaires de Rennes, 2005, p. 44. 
une connaissance technique du corps, sa mise en condition (si ce n'est sa transformation) et l'adoption de pratiques pornographiques: «Utilisez les sexy toys! » (Isa, juillet 2003), «Et si je lui faisais un striptease?» (Bien dans ma vie!, été 2005). « Avez-vous le corps de ses envies? » demande Jeune et Jolie (mars 2007).

Soyez audacieuses! Distinguez-vous ! N'hésitez à adopter le perçage, le tatouage et même la scarification (Adorable, novembre 2005). Puisque ces pratiques «font maintenant sensation auprès des femmes », faites donc comme les autres, soyez de votre temps, laisse entendre le magazine! C'est la quadrature du cercle : il faut [229] réussir à se démarquer des autres tout en faisant comme tout le monde!

Adoptez d'autres positions sexuelles, apprenez à aimer les actes sexuels vus dans la pornographie, amusez-vous avec les jouets sexuels, vous connaîtrez un épanouissement sexuel et, par conséquent, un épanouissement personnel, prescrivent les magazines pour filles. L'époque, on le voit bien, est à l'ordonnance de normes à suivre.

Les magazines féminins racolent pour les sex-shops, font la promotion de leurs gadgets... Dans son numéro de novembre-décembre 2005, Jalouse offre à ses lectrices un vibromasseur. Selon le directeur du magazine, «vendre un vibromasseur avec Jalouse correspond parfaitement à sa vocation avant-gardiste ».

Dans ces magazines, l'émancipation et l'autonomie féminines se réduisent à l'accomplissement sexuel performatif individuel et à l'adoption de codes pornographiques.

$\grave{A}$ lire les magazines féminins, qu'ils soient pour adolescentes ou pour femmes, on ne peut pas être surpris par la croissance de la consommation de pornographie par les femmes. C'est ce qui est nouveau par rapport à ce qui se pratiquait voici vingt ans, où cette consommation était essentiellement masculine. 


\section{La publicité « porno chic»}

\section{Retour à la table des matières}

Luxe et sexe font bon ménage. Saphisme, sadomasochisme, viol collectif, fétichisme, zoophilie, etc., sont des thèmes exploités dans la communication publicitaire.

Le «porno chic» désigne une pratique publicitaire qui puise son inspiration directement dans la pornographie. Le but principal de cette publicité, outre le fait qu'elle visait à élargir la clientèle, est de retenir l'attention du public et d'influencer son opinion à l'égard de la marque. La stratégie du « porno chic» des grandes marques de luxe a pour objectif de susciter un désir chez le consommateur tout en lui faisant mémoriser la marque, ce pourquoi la provocation est très utile. «En impliquant fortement le consommateur, le shockvertising [publicité provocatrice] garantit la remarquabilité de l'annonce et augmente son taux de [230] mémorisation de manière substantielle ${ }^{3}$. » Ce type de stratégie de communication peut s'avérer très efficace. "Dans un environnement publicitaire encombré, un contenu publicitaire choquant assure au message d'être entendu », attestent des chercheurs 4.

La « vogue sex-shop de la publicité » a été la marque de commerce de groupes comme Gaultier, Helmut Lang et Mugler ${ }^{5}$. Les marques de luxe Gucci, Versace, Dior ou Vuitton, puis celles de prêt-à-porter comme Eram et American Apparel, ont à leur tour adopté les codes de la pornographie pour publiciser leurs produits. Il est de bon ton de montrer une femme dénudée en position de soumission à un homme

3 Gilles Lugrin, Âmes sensibles s'abstenir: entre surenchère homosexuelle et "glam trash », la polémique du porno chic, ComAnalysis, $n^{\circ}$ 25, septembre 2001. Site consulté le 3 août 2002, http ://www.comanalysis.ch/ComAnalysis/Publication25.htm.

4 Darren W. Dahl, Kristina D. Frankenberger et Rajesh V. Manchanda, « Does it pay to shock? Reactions to shocking and non-shocking and content among University students », Journal of Advertising Research, vol. 43, $n^{\circ} 3,2003$, p. 268280.

5 Xavier Deleu, Le consensus pornographique, Paris, Mango, 2002, p. 27. 
(Weston). Des lesbiennes se pourlèchent pour un sac à main Ungaro. Un homme offre une bague Natan, la femme ouvre alors les jambes pour montrer sa disponibilité sexuelle; le bijou est décrit comme « la première télécommande jamais inventée ».

Le «pornographisme » de la publicité n'utilise pas que la nudité féminine, ni ne pratique que l'exacerbation de la féminité, il met fondamentalement en scène la soumission des femmes et leur asservissement sexuel. Le produit promu est associé à l'accès sexuel aux femmes. Cette objectivation sexiste érotise également l'agression sexuelle. La violence pornographique et ses symboles sont repris et mis au goût du jour. Les marins de Minute Maid se livrent à un gangbang. Le sadomasochisme, le fétichisme et le bondage sont à l'honneur. Cuir, latex, skaii, fessée, flagellation, menottes, des cuirasses métalliques aux ceintures de chasteté, les codes pornographiques sadomasochistes ont envahi la publicité. La zoophilie est banalisée. La modèle de Kookai est en position de levrette devant un mouton, parce qu'elle a « envie d'un pull ». Chez Sisley, une femme presse les pis d'une vache, dont le jet couvre sa figure, rappelant l'éjaculation faciale du cinéma $X$. Le tourisme sexuel y trouve également son compte. Une publicité de Thalys, faisant la [231] promotion, à l'époque des fêtes de Noël, d'un voyage en train pour Amsterdam, montre un Père Noël touriste devant une vitrine où un renne prostitué attend sa visite.

Le « porno chic » joue sur tous les tabous, y compris sur celui de la pédophilie: des gamines utilisées comme mannequins sont agenouillées, jambes écartées, seins dénudés (Sisley), léchant une sucette (Lee) ou tenant un ourson («Arrêtez de jouer tout seul ! » assène Goa).

Des fesses de femmes pour vanter un photocopieur, des seins pour vendre du parfum, des jambes pour les automobiles, des blondes inintelligentes pour de la bière ou d'autres produits... la publicité sexiste existe depuis longtemps. Elle a, par contre, été amplifiée de façon radicale par l'adoption des codes pornographiques. 


\section{La mode hypersexualisée}

\section{Retour à la table des matières}

«À quoi reconnaît-on une prostituée?», demande Florence Montreynaud 6 . Pour attirer les clients, les personnes prostituées s'habillent de façon provocante, se donnent à voir, font des gestes et adoptent des postures «obscènes». Elles se vêtent de façon à montrer une disponibilité sexuelle. Désormais, les repères sont brouillés, souligne l'historienne. Ce qui faisait mauvais genre auparavant s'est non seulement banalisé, mais a subi une inversion. La haute couture, analyse-t-elle, a rattrapé et même dépassé le style "prostitué », elle semble s'être donné le mot d'ordre de "faire plus pute que les putes». Les créateurs «se surpassent dans l'exhibitionnisme concurrençant sérieusement les panoplies de sex-shop $7 »$. Or, cette haute couture influence de façon importante les tendances du prêt-à-porter: jupe, blouse et débardeur du type "écolière », bas filet, souliers plateformes, talons aiguille, cuissardes, G-string, thong, etc.

Le conditionnement des jeunes filles au port de vêtements sexy illustre clairement la nature commerciale de cette pornographisation. Vers la fin des années quatre-vingt-dix, le string et le thong sont devenus les nouveaux must. Les filles âgées de treize à dix-sept [232] ans auraient dépensé, en 2003, près de 152 millions de dollars américains pour l'achat de string ${ }^{8}$. La Senza Girls a même mis en marché des soutiens-gorge rembourrés pour la taille 30AA. Ce type de mode impose très tôt aux jeunes filles un rôle - « charmer, plaire et sédui-

6 Florence Montreynaud, Amours à vendre, les dessous de la prostitution, Paris, Glénat, 1993, p. 44-46.

7 Ibid., p. 48.

8 Pamela Paul, Pornified. How Pornography is Transforming our Lives, our Relationships, and our Families, New York, Times Book, 2005, p. 184. 
re» - de lolita et de bimbo (style ultra-féminin), tout en leur inculquant le message : leur valeur se mesure à leur sex-appeal ${ }^{9}$.

Proies des marchands de la mode, des fillettes de sept, de neuf ou de onze ans apprennent à séduire par la mise en valeur sexuelle de leur être. Elles sont transformées en nymphettes et en mini-femmes fatales. Elles sont métamorphosées en objets de désir, en petites femmes sexy, alors qu'elles sont des fillettes et qu'elles n'ont pas encore les moyens d'être sujets de désir. Elles apprennent à dépendre du regard de l'autre pour exister. Les adultes qui abhorrent les pédophiles donnent pourtant à voir leurs enfants comme des objets sexuels. Et les jeunes filles se forgent une idée de la sexualité centrée sur la séduction et la consommation.

La sexualisation précoce des fillettes n'est pas une chose nouvelle 10. Ce qui est nouveau, c'est sa popularisation et son universalisation. La Dr Franciska Baltzer, pédiatre à l'Hôpital pour enfants de Montréal, rapporte à ce propos: «En 1983, à Sainte Justine, il était clair, pour nous, que les filles qui portaient des vêtements sexy à six ans ou à sept ans étaient des victimes d'abus sexuels. Aujourd'hui, rien n'est moins sûr étant donné que ce sont ces vêtements qui sont disponibles dans les magasins $11 »$, leur port étant désormais généralisé.

S'il est vrai que, traditionnellement, la petite fille était sexualisée au moyen de vêtements comme des robes, ce qui exigeait d'elle [233] un comportement approprié et une tenue « décente», avec toutes ses contraintes, la sexualisation contemporaine a donné naissance à un terme, l'« hypersexualisation », qui tente de rendre compte de la radicalité et de l'approfondissement du phénomène. Ce terme désigne l'excès de sexualisation ou son aggravation. Il renvoie également à la

9 Natasha et Pierrette Bouchard, La sexualisation précoce des filles peut accrốtre leur vulnérabilité, Sisyphe, 18 avril 2007, [site consulté le 17 septembre 2007] http ://sisyphe.org/imprimer/php3 ?id_article=917.

10 Voir à ce propos le chapitre II de Richard Poulin, Enfances dévastées. L'enfer de la prostitution, tome I, Ottawa, L'Interligne, 2007 : «Des lupanars antiques à l'organisation contemporaine de la prostitution des enfants », p. 50-79.

11 Franziska Baltzer, Sexualisation précoce des adolescent-es et abus sexuels, Sisyphe, 13 avril 2007, [site consulté le 19 janvier 2008], http ://sisyphe.org/article.php3 ?id_article=2073. 
notion de sexualisation précoce, laquelle est définie par le fait que les jeunes filles «sont poussées socialement à adopter des attitudes et des comportements de "petites femmes sexy" 12 ».

Aujourd'hui, les enfants baignent dans la sexualité adulte. Ils consomment très jeunes de la pornographie, tandis que les magazines pour filles ne cessent de traiter du sexe et de la séduction, comme si l'univers des lectrices ne devait se limiter qu'à ces questions et à celles des produits à acquérir pour mieux devenir des filles sexy et, par le fait même, tellement intéressantes que tous voudront les connaître et s'en faire des amies. Plaire devient synonyme d'« être sexy». Plaire passe par une féminisation adulte des jeunes filles et par leur objectivation sexuelle. On constate une entrée active dans une vie sexuelle de plus en plus hâtive ${ }^{13}$.

Toutefois, cette sexualisation précoce est parallèle et étroitement combinée avec un phénomène plus général, celui d'une société ellemême hypersexualisée, où le corps féminin est chosifié et morcelé et où la valeur des femmes est réduite à leurs attributs physiques et à leur capacité de plaire et de séduire. Celle également d'une société de l'extimité ${ }^{14}$, c'est-à-dire de l'intimité [234] surexposée dans la sphère publique. Celle d'une société axée sur la performance sexuelle. Celle enfin d'une société normative faisant l'éloge de la jeunesse, ce qui a généré une tendance au tout-jeunesse et au toujours-jeune des

12 Pierrette Bouchard, Consentantes? Hypersexualisation et violences sexuelles, Rimouski, CALACS de Rimouski, 2007, p. 6.

13 Les données révèlent que, chez les personnes âgées de 15 à 29 ans au moment de L'enquête sociale et de santé 1988 , ce sont $15 \%$ des répondant-es qui ont eu leur première relation sexuelle avec pénétration avant 15 ans, alors que cette proportion tombe à $8 \%$ chez les $30-39$ ans, $4 \%$ chez les $40-49$ ans et $3 \%$ chez les 50 59 ans. Institut de la statistique du Québec, Enquête sociale et de santé 1998, Québec, Les Publications du Québec, 2000, p. 206. Une étude récente de Statistique Canada, basée sur les résultats de l'Enquête longitudinale nationale sur les enfants et les jeunes (cycles de 1998-1999 et de 2000-2001), indique que 22\% des Québécoises âgées de 14 ou 15 ans affirment avoir déjà eu des relations sexuelles tandis que $17 \%$ des Québécois de sexe masculin répondent de la même manière. Didier Garriguet, «Relations sexuelles précoces», Rapports sur la santé, vol. 16, n 3, Ottawa, Statistique Canada, mai 2005, p. 11-21.

14 Concept repris de Serge Tisseron, L'intimité surexposée, Paris, Hachette Littératures, 2001. 
moeurs sociales ${ }^{15}$. Les enfants se comportent comme des adolescents, les adolescents comme des adultes, et nombre d'adultes sont en crise d'adolescence... Il y a non seulement perte des repères intergénérationnels, mais également brouillage des rôles sociaux. Le tout-jeunesse est à l'oeuvre dans les industries du sexe depuis un bon moment déjà.

\section{La sexualisation publique et la pornographisation}

\section{Retour à la table des matières}

L'industrie de la pornographie se caractérise, entre autres, par le fait qu'elle exploite des jeunes filles. Cela ne concerne pas uniquement la pornographie dite pédophile, qui est criminelle, mais également la pornographie générale, disponible et facilement accessible. Celle-ci capitalise en effet sur le fantasme de la Lolita. En tapant « Lolita porn », nous avons obtenu 1850000 résultats. Pour « teen porn», le nombre d'entrée s'élève à 12700000 . Pour «preteen porn », c'est-àdire des fillettes âgées de moins de treize ans, nous obtenons 1500000 entrées. Cela donne une idée de l'importance de la pornographie usant et abusant d'enfants ou de pseudo-enfants dans le cas des adolescentes (teenagers), qui peuvent être tout aussi bien âgées de dix-huit ou de dix-neuf ans que de treize à dix-sept ans.

La pornographie n'est qu'un aspect d'un problème plus large sur lequel elle agit, celui d'une société où une représentation sexualisée des jeunes filles et femmes sert de plus en plus à vendre tout et n'importe quoi, des parfums aux vêtements. Nous sommes bombardé-es par des images de jeunes femmes sexualisées... Et sexualisées de plus en plus jeunes.

Le concept de pornographisation de la culture permet de mettre en évidence deux éléments entrelacés: d'une part, l'étendue de l'influence de la pornographie dans les systèmes de représentation et de communication, et d'autre part, les normes adoptées et promues, [235] découlant de la pornographie, qui participent à forger les rôles

15 Agathe Fourgnaud, La confusion des rôles. Les toujours-jeunes et les déjà-vieux, Paris, Lattès, 1999. 
sexuels, lesquels conditionnent les relations entre les hommes et les femmes. Ce concept réfère donc au fait que les images sexuellement explicites et sexistes sont désormais largement répandues et que ce processus ne cesse de se développer et de prendre de l'ampleur. Il réfère également au fait que l'époque n'est plus à la suggestion mais à l'exhibition et à l'ordonnance de normes à suivre.

\section{La tyrannie du nouvel ordre sexuel}

\section{Retour à la table des matières}

L'envahissement pornographique s'est traduit par la mise en place d'un nouvel ordre sexuel. Les magazines pour femmes et pour adolescentes multiplient les dossiers racoleurs: «Poser nu, pourquoi pas vous? ", "Poser pour Playboy, oui c'est possible» (Le Mag des castings, juillet-août 2005), «Fantasmes, tabous, j'ose tout» (Bien dans ma vie!, été 2005), "Chéri! on se fait notre cinéma $X »(O H$ ! printemps 2008), «Cinéma $X$ : une histoire chaude» (Adorable, juillet 2005). Les tests du type «Quelle bête de sexe êtes-vous? » ou encore «Êtes-vous une véritable braise ou un vrai glaçon? 16 » sont désormais communs et récurrents. Suivent les conseils de «journalistes » à la fois normatifs et prosélytes.

Cette promotion de la pornographie s'accompagne d'une banalisation de la prostitution. Par exemple, la livraison de juillet 2005 du magazine Adorable fait l'éloge des gadgets sexuels trouvés en sex-shop, propose une histoire du cinéma $X$, conseille le sexe express accompagné d'« une panoplie de jouets sexuels» et défend l'idée que les prostituées et les vedettes du porno hard font un travail comme un autre, qui doit être valorisé ${ }^{17}$. Ces magazines voués à une clientèle féminine

16 Des menottes et une sorte de ceinture de «chasteté » en métal fermé par un cadenas sur un corps féminin illustrent ce dernier test proposé par Le Mag des castings (juillet-août 2005).

17 Le groupe Genex Communications publie le magazine Adorable. Ce groupe québécois contrôle des stations de radio, un studio de production, un réseau d'affichage, un club de hockey de la Ligue nord-américaine et une compagnie multimédia. 
jeune, souvent adolescente, ne sont pas les seuls à faire la promotion des «métiers» sexuels. Le magazine québécois, Jobboom, « un chef de file du recrutement [236] en ligne au Canada et un spécialiste du marché de l'emploi », qui offre «des conseils carrière et de l'information sur le monde du travail», a publié, en juillet 2007, un dossier sur les industries et les «métiers» du sexe.

Ces phénomènes se conjuguant, le recrutement pour ces industries s'en trouve facilité. C'est l'un des effets notables, mais souvent passés sous silence, de la banalisation des industries du sexe à l'échelle mondiale. Par exemple, dans les pays de l'Est, où la prostitution, la pornographie et la traite à des fins d'exploitation sexuelle ont explosé aux cours des années quatre-vingt-dix, des sondages ont révélé que près d'un sixième des élèves ukrainiennes percevaient la prostitution de façon positive, croyant que celle-ci n'était que luxe et plaisir à l'Ouest, et que le quart des filles de Moscou, élèves au secondaire, envisageaient de se prostituer 18 . Lorsque la prostitution et la pornographie apparaissent comme un métier comme un autre, elles deviennent des voies d'« avenir »... pour les jeunes femmes.

Les magazines féminins véhiculent un message sur la sexualité qui est loin d'être subtil. C'est, en substance, le suivant: presque tout le monde sauf vous a une vie sexuelle fascinante et variée. Adoptez d'autres positions sexuelles, apprenez à aimer les actes sexuels vus dans la pornographie, amusez-vous avec les gadgets sexuels et vous connaîtrez l'épanouissement sexuel.

Cette ordonnance en faveur d'un épanouissement tout au profit des industries du sexe adopte d'autres formes «transgressives» et «libératoires », que s'empressent de relayer les médias d'information. Par exemple, un « masturbothon » a été organisé à l'initiative d'un sexshop montréalais, Venez tels quels, la filiale québécoise de Come as you are, établi à Toronto depuis 1988. Il n'y a pas que les masturbateurs qui ont mis la main à la pâte, plusieurs artistes ont participé gratuitement à un spectacle-bénéfice dont les recettes ont été versées à

18 Sevgi O. Aral, Janet S. St Lawrence, Lilia Tikhonova, Emma Safarova, Kathleen A. Parker, Anna Shakarishvili, Caroline A. Ryan, «The social organization of commercial sex work in Moscow, Russia», Sexually Transmitted Diseases, vol. $30, n^{\circ} 1,2003$, p. 39-46. 
Stella, une association favorable au «travail du sexe». Ces deux activités ont permis à l'organisation Stella, qui reçoit déjà [237] d'importantes subventions gouvernementales, d'encaisser 25000 dollars. Pour Claire Thiboutot de Stella, « c'est très rare que des gens endossent notre cause au point d'organiser de $A$ à $Z$ une campagne de financement pour nous venir en aide. Mais, en même temps, c'est un peu normal. Nous partageons le même objectif que Venez tels quels, celui de démystifier la sexualité $19 »$. En effet, Stella poursuit des objectifs conformes aux intérêts des industries du sexe, dont la décriminalisation de la prostitution en bordels et du proxénétisme. Il n'est donc pas surprenant qu'un sex-shop, partie prenante de l'industrie du sexe, trouve un intérêt à participer au financement d'un tel groupe et à renforcer son « combat ».

\section{La saturation sexuelle et sexiste de l'espace public}

\section{Retour à la table des matières}

Nombre de médias sont axés sur le « divertissement sexuel ». Il y a évidemment les magazines pour hommes, qu'ils soient pornographiques ou non, qui utilisent à foison le corps féminin. Mais il y a également les magazines féminins, dont les couvertures ressemblent souvent étrangement à celles des magazines pour hommes. Au point que les distinctions s'estompent. Aussi n'est-il pas surprenant que lors d'un colloque sur la pornographie tenu en avril 2008 à l'Université d'Ottawa, une affiche de promotion qui utilisait essentiellement des couvertures de magazines féminins et des publicités tirées desdits magazines ait pu sembler pornographique et se voir refuser le droit d'affichage dans certains édifices.

Les médias célèbrent le consumérisme. C'est particulièrement évident dans les magazines féminins établis de longue date, dont au moins la moitié des pages comportent des publicités, tandis qu'une autre partie assez importante n'est que promotion des produits de compagnies ayant acheté des espaces publicitaires. Évidemment, cette dernière

19 Émilie Dubreuil, « Sexe Masturbation », La Presse, 5 juin 2005. 
promotion ne serait pas de la publicité à proprement parler, elle n'est $q u^{\prime} \ll$ information », puisque ces médias ont vocation d'« informer »!

Le principal rôle des médias de masse, qui sont eux-mêmes une industrie visant la rentabilité, est de vendre un auditoire ou un lectorat aux publicitaires. En 2004, le PDG de TF1, la principale [238] chaîne de télévision française, a fait scandale en énonçant crûment le rôle des médias: «À la base, le métier de TF1, c'est d'aider Coca-Cola, par exemple, à vendre son produit [...] Pour qu'un message publicitaire soit perçu, il faut que le cerveau du téléspectateur soit disponible. Nos émissions ont pour vocation de le rendre disponible: c'est-à-dire de le divertir, de le détendre pour le préparer entre deux messages. Ce que nous vendons à Coca-Cola, c'est du temps de cerveau humain disponible 20 . »

Pour vendre "du temps de cerveau disponible», il s'agit non seulement de « divertir » les consommateurs potentiels, mais de cultiver la désirabilité des objets de consommation. Françoise Brune voit en cela une implication lourde de sens: «Il n'y a de désir que d'objet et donc [...] il faut se faire objet pour être désiré 21 . " La mise en scène de la femme-objet par la publicité ne doit donc rien au hasard. Consumérisme et marchandisation des corps, surtout des corps féminins, vont de pair.

\section{De l'intimité surexposée}

\section{Retour à la table des matières}

Depuis le milieu des années 1990, nos sociétés se caractérisent également par une surexposition publique de l'intimité 22 . Ce phénomène résulte d'un double processus : la privatisation de l'espace public et la publicisation de l'espace privé. Nous sommes à «l'âge de l'individu quelconque, c'est-à-dire un âge où n'importe qui doit s'exposer dans

20 Cité par Marie Bénilde, On achève bien les cerveaux. La publicité et les médias, Paris, Raisons d'agir, 2007, p. 19.

21 Françoise Brune, Le bonheur conforme, Paris, Gallimard, 1985, p. 241.

22 Tisseron, op. cit. 
l'action personnelle afin de produire et montrer sa propre existence $23 »$. L'identité personnelle réussie exige une visibilité sociale et, en retour, la visibilité sociale est la preuve du succès de la quête identitaire individualisée. La singularité doit devenir spectacle pour s'avérer «réelle» ou «vraie». Les gens meurent d'envie de passer sur les écrans, « d'accorder une valeur à leur vie 24 », afin que celle-ci ne s'abîme pas dans le néant, dans le non-être. Nous pouvons donc comprendre, dans de telles conditions, [239] le succès des télévérités. Dans ces émissions, l'individu quelconque se réalise en tant qu'individu, en pensant échapper à sa condition d'anonyme. Pour cela, il doit accepter de se dévoiler, de donner un accès public à son intimité, tant psychique que physique. Les jeunes gens de Loft Story acceptent de se livrer à leur toilette ou à leurs ébats sexuels devant les caméras.

Les années quatre-vingt-dix ont fait du corps des femmes un temple du marché, l'objet de transactions et un support commercial. Leur autonomie plus grande, une conquête essentielle du mouvement féministe, a été transformée, au fil du triomphe des relations marchandes et du néolibéralisme, en une soumission accentuée aux plaisirs sexuels masculins. C'est l'ère des légalisations du proxénétisme et de la prostitution des jeunes femmes en bordels et dans des zones dites « de tolérance », en Europe de l'Ouest et dans le Pacifique-Sud. C'est également l'époque où explose la production et la consommation pornographique. L'injonction « libératrice » est désormais individualisée et non plus collective. Elle a réintroduit par la porte arrière ce qui avait été chassé devant, l'obligation d'un lourd entretien féminin sexualisé des corps, lequel est devenu très onéreux : diététique, cosmétique, exhibition vestimentaire, centre de conditionnement physique, chirurgie plastique, etc. Les ventes de lingerie féminine progressent de dix pour cent par an depuis les années quatre-vingt. L'essor de la chirurgie plastique est phénoménal: «Le nombre d'interventions réalisées mondialement a grimpé vertigineusement 25 . » La juvénilité obligée du corps féminin l'infantilise : nymphoplastie (opération « esthétique » des peti-

23 Alain Ehrenberg, Le culte de la performance, Paris, Hachette Littérature, 1991, p. 279.

24 Ibid., p. 280.

25 Angelika Taschen (dir.), La chirurgie esthétique, Köln, Taschen, 2005, p. 10. 
tes lèvres du vagin), resserrement des parois vaginales, épilation totale des poils pubiens, etc.

Les nouvelles prescriptions sont corporelles. Le corps féminin transformé et mutilé est plus que jamais une surface d'inscription de l'idéologie dominante, à la fois bourgeoise et sexiste. Le corps est désormais traité comme une propriété individuelle, dont chacun est responsable. Ses métamorphoses aggravées sont paradigmatiques de la beauté et de la séduction. Le contrôle individuel sur le corps suggère un contrôle sur la vie, laquelle sera par conséquent [240] épanouie. Plus le corps est moulé et exhibé, plus il est artificiellement construit et dépouillé de sa naturalité, plus il est.

Dans la nouvelle mouture du capitalisme, le contrôle de soi est la condition à la vente de soi, laquelle est elle-même une condition de la réussite sociale. À l'ère du néolibéralisme, la « revendication de ne pas être une chose, un instrument, manipulable et marchandisable, serait passéiste et non une condition de dignité du sujet», assène Véronique Guienne 26. L'apparence est décisive dans le travail sur soi pour sa propre mise en valeur.

Les régressions sont à la fois symboliques - retour à la femmeobjet 27 - et tangibles : exploitation sans précédent des corps féminins par les industries du sexe, reculs sur le droit à l'avortement, pauvretés et inégalités accrues à l'échelle mondiale, etc.

Les nouvelles prescriptions sont également sexuelles. Performatives, elles s'inspirent de la pornographie et de ses codes, devenus le nouveau manuel de la libération sexuelle. En 1981, est « découvert» le point $G$, cette zone intravaginale ultrasensible au-dessus de l'os pubien. Cette trouvaille débouche sur une optimisation des performances coïtales et l'obligation des jouissances multiples. L'injonction de jouir, une preuve de la réussite sexuelle, est désormais une condition de la santé et de l'équilibre mental. Si l'« eldorado orgasmique» est à la portée de toutes, il n'en reste pas moins que les jeunes femmes

26 Véronique Guienne, « Savoir, se vendre : qualité sociale et disqualification sociale », Cahiers de recherche sociologique, $n^{\circ} 43$, janvier 2007, p. 13.

27 Christine Détrez, et Anne Simon, À leur corps défendant. Les femmes à l'épreuve du nouvel ordre moral, Paris, Seuil, 2006, p. 12. 
consultent majoritairement pour leur « frigidité » réelle ou supposée et pour la douleur lors des rapports sexuels. Dans les cabinets, « les plaintes les plus fréquentes en matière de sexualité viennent des adolescentes et des femmes de moins de trente ans », constate la gynécologue Anne de Kervasdoué 28 . Plus de $50 \%$ trouvent les rapports douloureux. En outre, est constatée chez les filles une multiplication de pathologies où le corps dit non à la place de la tête: mycoses à répétition, inflammations, douleurs inhabituelles.

Cette biopolitique 29 inédite du corps impose un contrôle intériorisé contraignant pour tous, mais avant tout pour les femmes [241] qui sont ses cibles charnelles privilégiées. «Plutôt qu'à une disparition des contraintes, on assiste à une intériorisation des maîtrises et des surveillances », explique Philippe Perrot ${ }^{30}$. Pour être belle, une femme doit être jeune et le rester 31 .

«Le jeunisme est un ressort idéologique majeur des années 1980 32. » On le voit en oeuvre partout. La norme dans la pornographie, la publicité et la mode (notamment avec son utilisation de mannequins très jeunes) est largement «adocentriste». Mais si les jeunes, particulièrement les jeunes femmes et les adolescentes, sont parmi les principales cibles des vendeurs de biens de consommation, ils sont également des biens de plus en plus consommables. On constate à l'échelle mondiale un rajeunissement des personnes prostituées et une explosion de la pornographie pseudo-infantile et infantile. On constate également une sexualisation précoce des filles, imprégnées de références sexuelles adultes. Les garçons, s'ils n'adoptent pas le style vestimen-

28 Dans Blandine Kriegel, La violence à la télévision, Paris, PUF, 2003.

29 Concept développé par Michel Foucault, Histoire de la sexualité tome 1. La volonté de savoir, France, Éditions Gallimard, 1976.

30 Philippe Perrot, Le travail des apparences. Le corps féminin, XVIIIe-XIXe siècle, Paris, Seuil, 1984, pp. 206-207.

31 Jean-Claude Kaufmann dans Corps de femmes, regards d'hommes. Sociologie des seins nus, Paris, Nathan, 1998, a montré la force de l'ostracisme encouru par les personnes âgées dans le lieu de liberté apparente et de la tolérance affichée, la plage.

32 François Cusset, La décennie. Le grand cauchemar des années 1980, Paris, La Découverte, 2008, p. 280. 
taire pimp, s'attendent à ce que les filles reproduisent les attitudes et les actes consommés dans la pornographie, ainsi que les pratiques corporelles qui lui sont liées comme l'épilation. Un pubis non épilé provoque la rupture, ont témoigné des jeunes femmes. Les contraintes ont changé de nature. La nouvelle morale sexuelle, tout aussi normative que l'ancienne, impose un nouvel ordre sexuel tyrannique, lequel se traduit dans des normes corporelles et des rapports sexuels focalisés sur le plaisir masculin et la génitalité.

\section{L'influence sociale pornographique}

Retour à la table des matières

La pornographie est un lieu de cristallisation idéologique où s'exprime la philosophie d'une époque 33 et qui, en retour, agit sur l'époque en fortifiant certains de ses traits. La pornographie emblématise les corps féminins comme autant d'objets-fantasmes mis au [242) service sexuel fantasmagorique des hommes et exploités réellement par les industries du sexe. Elle féminise les enfants, leur prêtant une maturité sexuelle d'adultes, tout en infantilisant les femmes.

La mise en scène de la sensualité d'adolescentes et de pseudoadolescentes - présentées comme tentatrices, séductrices et corruptrices - excite de nombreux hommes qui se masturbent en les regardant sur écrans interposés.

Ce que nous avons nommé « la pédophilisation 34 » rend compte à la fois du jeunisme comme ressort idéologique qui s'est imposé à partir des années 1980, du rajeunissement du recrutement par les industries du sexe - au Canada, l'âge moyen de recrutement dans la prostitution tourne autour de 14 ans -, de sa mise en scène par la pornographie et de «l'adocentrisme » de ces représentations. Il rend également compte des techniques d'infantilisation employées par l'industrie. Cepen-

33 Concept repris de l'analyse de la littérature populaire d'Antonio Gramsci, « Littérature populaire », OEuvres choisies, Paris, Édition Sociales, 1959.

34 Richard Poulin avec la collaboration de Mélanie Claude, Pornographie et hypersexualisation, Enfances dévastées, tome II, Ottawa, L'Interligne, 2008. 
dant, le rajeunissement constaté n'est pas que la conséquence des modalités actuelles de la production des industries du sexe, il joue également dans la consommation. Désormais, on consomme très jeune, à l'âge moyen de douze ans pour les garçons et de treize ans pour les filles. La pornographie devient le principal lieu d'« éducation» sexuelle et un modèle pour les relations sexuelles. Plus les jeunes consomment tôt, plus leurs désirs, leurs fantasmes et leurs pratiques s'inspirent des codes pornographiques. Plus ils consomment jeunes, plus leurs corps sont modifiés, tatoués et percés. Plus ils consomment jeunes, plus ils demandent à leur partenaire de reproduire les actes sexuels qu'ils ont vus. Plus ils consomment jeunes, plus ils consomment avec régularité et fréquence. Plus ils consomment jeunes, plus ils sont anxieux quant à leur corps et à leurs capacités physiques ${ }^{35}$. Il ressort également de la recherche que la consommation de pornographie par les jeunes filles affecte leur estime de soi. Par ailleurs, plus l'estime de soi est faible, plus les jeunes filles sont précocement actives sexuellement. L'enquête de Statistique Canada sur la santé montrait que « les filles dont l'image de soi était faible à l'âge de douze ou treize ans étaient plus susceptibles que celles qui avaient une [243] forte image de soi de déclarer, dès l'âge de quatorze ou quinze ans, avoir déjà eu des relations sexuelles 36 ». Alors que 10,9\% des filles qui affichent une bonne estime de soi déclarent avoir eu des relations sexuelles avant quinze ans, cette proportion est presque deux fois supérieure $(19,4 \%)$ chez celles qui affichent une piètre estime de soi 37 . Plus elles sont actives précocement, plus elles sont perçues comme des « salopes», ce qui n'est certes pas le cas des gars.

Bref, plus la consommation se fait jeune, plus elle a de conséquences tangibles et durables.

\section{Ibid.}

36 Statistique Canada, Les relations sexuelles précoces, 3 mai 2005, [site consulté le 15 mai 2005], http ://wwwstatcan.ca/Daily/Français/05053/q05053a.htm.

37 L'enquête sociale et de santé auprès des enfants et des adolescents québécois 1999 (Institut de la statistique du Québec, op. cit.) indique que $61 \%$ des filles de seize ans qui ont fréquenté un garçon dans l'année qui a précédé le sondage et qui avaient une faible estime de soi ont subi de la violence. Chez les filles qui affirmaient avoir une estime d'elles-mêmes élevée, ce taux se situait à la moitié, soit $30 \%$. 
Les femmes, les filles et même les fillettes maintenant sont poussées à l'exhibition. Ce devoir de paraître, qui exige beauté et jeunesse, est déguisé au droit au bien-être. Le corps doit être lisse, désirable, désirant et performant. Il est en même temps morcelé, ses parties sont offertes tour à tour, ce qui est particulièrement évident dans la publicité, et davantage dans la pornographie. La partie est préférée au tout et l'érotisme masculin contemporain se caractérise par un « fétichisme polymorphe», du sein en passant par les fesses jusqu'au pied. Par ailleurs, « la loupe portée sur tous les détails conduit d'abord à écarter les corps réels du corps idéal, les corps vécus du corps rêvé ${ }^{38}$. " Le corps féminin réel, malgré tous les efforts qui lui sont consacrés, déçoit fatalement, particulièrement les hommes qui ont commencé à consommer de la pornographie très jeunes.

\section{Pouvoirs}

\section{Retour à la table des matières}

Les représentations des corps et les valeurs qu'elles induisent, le travail incessant de gestion des apparences pour se conformer à cet idéal, reproduisent à leur échelle les pouvoirs de la structure sociale. L'assise de la domination «passe par la maîtrise des usages [244] du corps et l'imposition de ses normes 39 ». Les normes qui se sont imposées sont fortement corrélées historiquement à l'ascension de la bourgeoisie, puis à sa victoire 40 . La domination masculine impose non seulement une division sexiste du travail et une essentialisation des rôles - à l'homme la raison et la sphère publique, à la femme la procréation, les émotions, le travail des apparences et la sphère privée -, mais également une maîtrise du corps féminin, laquelle est intériorisée par les principales concernées, les dominées ${ }^{41}$. Si la domination masculine

38 Perrot, op. cit., p. 67.

39 Christine Détrez, La construction sociale du corps, Paris, Seuil, 2002, p. 173.

40 Voir entre autres Foucault, op. cit. ; Georges Vigarello, Le corps redressé, Paris, Delarge, 2001 ; Alain Corbin, Le miasme et la jonquille, Paris, Aubier, 1982.

41 Pierre Bourdieu, La domination masculine, Paris, Seuil, 1998. 
vêt les femmes - du voile à la haute couture -, elle les dévêt également dans la publicité, la pornographie et ailleurs. Ce sont les regards des hommes qui décident des corps des femmes ${ }^{42}$. Les corps sont des enjeux de pouvoirs, tout en étant leur symbolisation. L'époque actuelle inscrit systématiquement et massivement dans les corps les disparités sociales entre les sexes et les générations. Dans ce cadre, la liberté sexuelle libérale " permet aux plus forts, plus riches, plus cyniques de cautionner leurs désirs criminels au détriment des plus faibles ou des plus pauvres $43 »$. L'argent-roi donne accès aux femmes et aux filles partout dans le monde ainsi que sur tous les supports médiatiques, tout en légitimant leur exploitation sexuelle.

Cette domination trouve une forme d'expression ultime dans les productions pornographiques qui pèsent considérablement sur les représentations collectives dominantes qu'elles «pédophilisent». Dans son témoignage, l'ex-hardeuse Raffaëla Anderson raconte: «Elle termine enfin de me maquiller. Quand je vois ce que ça donne, je suis déçue. Je ressemble à une gamine de douze ans ${ }^{44}$. »

Le préadolescent et l'adolescent d'aujourd'hui sont gavés de pornographie. Ils sont accoutumés à une vision sexiste des rôles sexuels. Leur imaginaire sexuel est nourri par les produits de cette [245] industrie et, puisque le sentiment et la tendresse sont tabous dans la pornographie, puisque le sexe mécanique est valorisé, l'objectivation et l'instrumentalisation des femmes et des filles s'en trouvent socialement renforcées. Ils voient dans les filles de leur âge des objets sexuels potentiels. Les garçons affichent des conduites de contrôle sexuel, assure le psychothérapeute James Wright. Ces attitudes et les comportements qui en découlent commencent très tôt, habituellement à la fin de l'école primaire, et sont étroitement imbriqués à leur perception de la masculinité 45 , laquelle est déterminée par l'environnement social, où la pornographie joue certainement un rôle.

42 Kaufmann, op. cit.

43 Dominique Folscheid, Sexe mécanique. La crise contemporaine de la sexualité, Paris, La Table Ronde, 2002, p. 14.

44 Raffaëla Anderson, Hard, Paris, Grasset, 2001, p. 17.

45 James E. Wright, The Sexualization of America's Kids and How to Stop It. New York, Lincoln, Shanghai, Writers Club Press, 2001. 
Une enquête canadienne, menée auprès de 3000 élèves de huit écoles secondaires de Montréal, Kingston et Toronto, a révélé que «trois élèves sur quatre se font harceler sexuellement par leurs pairs $46 »$. Dans une société où la sexualité, surtout celle des jeunes femmes, est un bien de consommation qui sert à vendre des marchandises et à exciter sexuellement les hommes, il n'apparaît pas étonnant que l'on constate des taux élevés de harcèlement et d'agression sexuels et que les cibles de ces agressions soient surtout des adolescentes.

Dans la pornographie, « la femme crie et jouit de la jouissance de l'homme 47 ». L'adéquation est parfaite entre l'homme qui veut et la femme qui accepte d'être à son service sexuel. "La femme doit apprendre à aimer son corps, afin de pouvoir donner du plaisir 48 . » Voilà peut-être le fin mot de l'histoire: la liberté c'est bien, mais à condition qu'elle profite aux hommes.

Mettre en valeur son corps pour faire plaisir aux garçons et aux hommes s'apprend tôt et, aujourd'hui, le corps des fillettes se forme en se conformant aux modèles dominants, qui sont de plus en plus pornographiques.

Fin du texte

46 Citée par Pierrette Bouchard, op. cit., p. 52.

47 Matthieu Dubost, La tentation pornographique, Ellipses, Paris, 2006, p. 66.

48 Détrez et Simon, op. cit., p. 245. 